\title{
Uso del ejercicio físico con fines profilácticos en el Adulto Mayor
}

Use of physical exercise for prophylactic purposes in the elderly

MSc. Eugenio Rodolfo González Pérez. ${ }^{1}$ \& Lic. José Díaz Valdés. ${ }^{2}$

DOI: https://doi.org/10.32/cienciadigital.v3i1.947

\begin{abstract}
.
A comprehensive search was conducted on the use of physical exercise applied to the elderly. We will briefly explain how physical exercise helps the elderly in terms of the health-disease process. The aim was to describe how physical exercise helps the older adult physically and emotionally. Reference was made to the group of older adults corresponding to the care area of the Martha Martínez Figuera Polyclinic South, where 137 people over 60 years of age were chosen, of whom 60 were women and 77 men. It is known that the elderly tend to be affected by most chronic diseases because they have a weakened immune system as they age, so the use of physical exercise should provide a solution in terms of improving the health of people of this age. This is how the concepts to be used and the methods used to finally understand how the use of physical
\end{abstract}

\section{Resumen.}

Se realizó una búsqueda exhaustiva del uso del ejercicio físico aplicado al Adulto Mayor. Se expondrá brevemente cómo ayuda el ejercicio físico al adulto mayor en cuanto al proceso salud-enfermedad. Se pretendió describir cómo ayuda el ejercicio físico al adulto mayor físicamente y emocionalmente. Se tomó como referencia al grupo del adulto mayor correspondiente al Área de atención del Policlínico Sur Martha Martínez Figuera, donde fueron escogidos 137 personas mayores de 60 años, de los cuales eran 60 mujeres y 77 hombres.Se utilizaron métodos grupales y técnicas de atención al adulto mayor tales como gimnasia rítmica, juegos y formas jugadas y gimnasia suave. Se conoce que el adulto mayor tiende a ser afectado por la mayoría de enfermedades crónicas al poseer un sistema inmune debilitado al paso de la edad, por lo que el uso del ejercicio

\footnotetext{
1 Facultad de Ciencias Médicas de Mayabeque, Departamento Cultura Física, Mayabeque, Cuba, ergonzales@infomed.sld.cu

${ }^{2}$ Facultad de Ciencias Médicas de Mayabeque, Mayabeque, Cuba, jdiaz@infomed.sld.cu
} 
exercise really helps the older adult were presented in a direct way. A retrospective longitudinal descriptive study was carried out to better understand the problem posed and its possible solution, in the universe taken from 137 people in the southern care area, that is, belonging to the Policlínico Sur Martha Martínez Figuera.

Keywords: Reflexology podal, arterial Hypertension. físico debe brindar una solución en cuanto al mejoramiento de la salud de las personas de esta edad. Así se expuso de manera directa los conceptos a utilizar y los métodos utilizados para entender finalmente cómo el uso del ejercicio físico ayuda realmente al adulto mayor. Se realizó un estudio descriptivo longitudinal retrospectivo para comprender mejor el problema planteado y su posible solución, en el universo tomado de 137 personas en el área de atención sur, es decir perteneciente al Policlínico Sur Martha Martínez Figuera.

Palabras claves: Reflexología podálica, Hipertensión arterial

\section{Introducción.}

El envejecimiento de la población es un fenómeno universal, enmarcado en la encrucijada del descenso de la tasa de natalidad y el aumento de la esperanza de vida por los adelantos médicos, sobre todo en los países de buen nivel de vida. En el año 2000 se elevó los años de la población mundial a un promedio de 65 años. ${ }^{[1]}$

El envejecimiento se asocia a una reducción de la capacidad aeróbica máxima y de la fuerza muscular, así como de la capacidad funcional en general, así podemos considerar que el ser humano evoluciona físicamente hasta los 20 años e involuciona claramente entre los 65-70 años.

El ejercicio es una necesidad corporal básica. El cuerpo humano está hecho para ser usado, de lo contrario se deteriora; si se renuncia al ejercicio el organismo funciona por debajo de sus posibilidades físicas por tanto se abandona de la vida. Un cuerpo que no se ejercita utiliza solo el $27 \%$ de la energía posible de que dispone, pero este bajo nivel de rendimiento puede incrementarse hasta $56 \%$ si se práctica regularmente ejercicios, este aumento de crecimiento orgánico podrá ser apreciado en todos los ámbitos de la vida. ${ }^{[2][3]}$

En general podemos resumir que la realización de ejercicios físicos de forma regular es una necesidad de todo ser humano ya que su influencia es una vía segura para evitar el deterioro orgánico, lograr una vida sana, retrasar los síntomas del envejecimiento, y evitar enfermedades ya que el ejercicio regular reduce el riesgo de enfermedades cardiacas, reduce la resistencia vascular periférica e incrementa la distensibilidad aórtica con lo cual disminuye la presión arterial y mejora el retorno venoso debido a que incrementa la fuerza, la resistencia y la eficacia del corazón; dependiendo del grado de esfuerzo y del tipo de ejercicio realizado, 
los músculos aumentan de tamaño, fuerza dureza, resistencia y flexibilidad, también mejoran los reflejos y la coordinación, así como las demás funciones vegetativas en general. ${ }^{[4]}$

La prolongación de la vida y la capacidad de trabajo de la persona de edad avanzada es uno de los problemas sociales más importantes en los que debe incidir el profesional de la Cultura Física en la lucha por la salud y la longevidad.

En una revisión en 2008 de estrategias de enriquecimiento para frenar o revertir el deterioro cognitivo concluyó que «la actividad física y el ejercicio aeróbico en particular, mejora las funciones cognitivas en adultos mayores». ${ }^{[5][6]}$

La práctica regular de un programa de ejercicio genera nuevas redes neuronales y mejora las funciones cognitivas al incrementar la plasticidad sináptica, el metabolismo y la circulación sanguínea cerebral. ${ }^{[7]}$ Mejora la capacidad intelectual y el rendimiento escolar en niños y adolescentes. En experimentos con ratones, el ejercicio promueve la función cognitiva a través de la desarrollo del hipocampo que dependen de aprendizaje espacial, y la mejora de la plasticidad sináptica y la neurogénesis adulta ${ }^{[8]}$ Además, la actividad física tiene efectos neuroprotectores en muchas enfermedades neurodegenerativas y neuromusculares. ${ }^{[9]}$ Por ejemplo, reduciría el riesgo de desarrollar demencia. ${ }^{[30]}$ Por otra parte, la evidencia anecdótica sugiere que el ejercicio regular puede revertir el daño cerebral inducida por el alcohol. ${ }^{[10][11]}$

Las posibilidades de por qué el ejercicio es beneficioso para el cerebro son los siguientes:

- Aumentando el flujo de sangre y oxígeno al cerebro.

- Aumentando los factores de crecimiento neuronales que ayudan a crear nuevas células nerviosas ${ }^{[12]}$ y promover la plasticidad sináptica. ${ }^{[13]}$ En una reciente investigación se comprobó que el ejercicio aumenta la expresión del factor neurotrófico derivado del cerebro BDNF, mediado por la hormona irisina. ${ }^{[14]}$

- Incremento de neurotransmisores cerebrales que ayudan a la cognición, como la dopamina, glutamato, noradrenalina y serotonina. ${ }^{[15]}$

La actividad física se cree que tiene otros efectos beneficiosos relacionados con la cognición ya que aumenta los niveles de factores de crecimiento nervioso, que soportan la supervivencia y el crecimiento de un número de células neuronales. ${ }^{[16]}$

\section{Desarrollo}

En 1965, el profesor Hayflick, cancerólogo de la escuela de medicina de la Universidad de Stanford (California), demostró que los filvoblastos (células del tejido intersticial) procedentes de un pulmón de feto humano sufrían, en promedio unas cincuenta divisiones y luego morían mientras que era solamente unos veinte las divisiones en el caso de los 
fibroblastos procedentes de un pulmón de adulto, concluía que el número de divisiones de una célula es inversamente proporcional a la edad del donante. ${ }^{[17][18]}$ Según Hayflick, el envejecimiento de un individuo con origen genético está determinado en el momento mismo de su concepción. El hombre "estaría en los últimos cuando su programa, inscrito en las moléculas de $\mathrm{ADN}$, tocase a su fin.

Es de destacar que ciertas sustancias tóxicas, virus radiaciones pueden alterar también el código genético y provocar en los tejidos lesiones características del envejecimiento. ${ }^{[19]}$

No obstante, parece que la existencia de esta programación celular, no es la única causa del envejecimiento humano, dado que, de todas maneras, el hombre no vive el suficiente tiempo para agotar el programa de su multiplicación celular.

\section{La teoría de la acumulación de errores de Orgel.}

En 1963, el doctor Orgel, del famoso Instituto Salk de California, ha mostrado a través de estudios bioquímicos o genéticos, que durante el envejecimiento se producen cada vez más errores en la síntesis de las proteínas nucleares ácidas. Parece que la célula vieja pierde de alguna forma la facultad de "discriminación", pudiendo, por ejemplo: en un 50\% de los casos utilizar sin razón un ácido aminado artificial proporcionado por el experimentador en lugar de su correspondiente ácido natural. ${ }^{[20][21]}$

Según Orgel, el programa genético estaría grabado ya no en una cinta magnética, sino en un disco, que, a fuerza de girar, se rayaría cada vez más.

La acumulación de errores resultante haría que al cabo de un tiempo la célula viva parece incapaz de comprender el mensaje transmitido, cometiendo errores y terminando por dejar de funcionar.

Pero entonces ¿cómo explicar los resultados de experimentos como el de la "parobiosis" u operación de los gatos siameses? Después de haber practicado suturas de vena a vena y arteria a arteria entre una rata de laboratorio y una vieja rata, que comparten así una sola circulación sanguínea (La sangre arterial bombeada por el corazón de la rata, joven pasa por el corazón de la rata vieja, riega sus tejidos, es oxigenada por sus pulmones y vuelve a la rata joven), se constata que la rata vieja vive más tiempo sin que por ello la longevidad de la rata joven haya disminuido. ${ }^{[22]}$

Es más significativo todavía el experimento realizado con cucarachas, que de joven tienen la facultad de regenerar las patas arrancadas, mientras que de mayor las pierden. Las viejas cucarachas con el riego sanguíneo de sus jóvenes hermanas siamesas, no solo viven más tiempo, sino que pueden así mismo regenerar sus patas. 
Se sabe también que en animales de sangre fría, que un descenso de la temperatura corporal incrementa la longevidad. De esta forma se logra multiplicar por diez la vida de reptiles haciendo bajar su temperatura sin, no obstante, trastornar las funciones de su organismo.

Por consiguiente, parece que un factor distinto del A.D.N. regulador, permite que una célula sobrepase sus límites. Este factor, sean hormonas una asociación de hormonas u otros productos del organismo todavía no identificados, actuaría como un elixir de juventud capaz de reducir el efecto de "bola de nieve" de los errores acumulados y de retrasar el desgaste del disco programador.

Se puede pensar que las dos teorías mencionados anteriormente no son forzosamente contradictorias si se postula que la potencialidad de los sistemas de reparación es la que está programada en las distintas especies. ${ }^{[23]}$

\section{Deterioro del tejido conjuntivo}

Definición considerada en otro tiempo como un tejido de "relleno" o de envoltura del cuerpo destinado a servir de sostén a los órganos a unirlos entre si y a la pared del cuerpo, pero también a separarlos (tejido intersticial), el tejido conjuntivo, elemento más importante de los músculos, de la piel, de los vasos sanguíneos, del miocardio, del epitelio pulmonar, está considerado hoy día por los biólogos como uno de los factores esenciales de las funciones más vitales.

\section{Composición:}

$>$ Elementos celulares: En forma de estrellas, unidos por finas prolongaciones citoplasmáticas (histiocitos, fibroblastos, fibrocitos)

- Fibras que aseguran:

- El sostén: Fibras colágenas (colágena rígida)

- La Elasticidad: Fibras elásticas (elastina flexible)

Estas dos clases de fibra se entrecruzan en todas las direcciones y constituyen una verdadera red en cuyas mallas se encuentran los leucocitos.

$>$ Gel Coloidal: Que es una sustancia compuesta por voluminosas moléculas de glucoproteínas que, por su mayor o menor polimerización, forma un "pegamento" más o menos compacto, más o menos hidrófilo a través del cual se efectúan los intercambios. ${ }^{[24]}$

\section{Conclusiones.}

- En el trabajo físico con adultos mayores no está permitido todo.

- Aun cuando el contenido de cada uno de los medios a utilizar no tiene nada de especial, si merecen ser adaptados a las exigencias de la edad y a las posibilidades funcionales y capacidad física de los participantes.

- Si se efectúa un ejercicio de forma moderada, el consumo de oxígeno aumenta gradualmente, y después de uno o dos minutos se estabiliza o se queda al mismo nivel 
durante todo el ejercicio, también se mantienen constantes otras funciones como por ejemplo la respiración, la frecuencia cardíaca y la producción de ácido láctico; esta condición está definida como estado estacionario.

- El ejercicio debe realizarse con una intensidad que no supere el estado estacionario por lo que es necesario cuidarse.

\section{Recomendaciones.}

En la atención al adulto mayor es necesario atender algunos aspectos pedagógicos metodológicos de los cuales depende el éxito en el desarrollo de las actividades, nos referimos a:

\section{Actitud Pedagógica}

D Ética del profesor: Es esencial una ética consecuente que transmita confianza, respeto profesional, mejore la empatía factores que condicionan una entrega total en la actividad.

$>$ Personalidad del profesor: Aparte de la competencia técnica es necesario que el profesor de actividades físicas posea los siguientes rasgos:

- La disponibilidad: Tomarse el tiempo necesario para observar, despertar el interés personal, facilitar los intercambios, interesar a los participantes, integrarles en el grupo, estimular la iniciativa y mantener el gusto por la actividad.

- La receptividad: Saber escuchar a la persona mayor para responder a sus necesidades, satisfacer sus motivaciones, fortalecerlas y crear otras nuevas.

- La vigilancia: Estar atento para inspirar seguridad y confianza y prevenir en los más audaces, los riesgos de accidentes que siempre pueden ocurrir.

\section{Referencias bibliográficas.}

Stampfer, M. J.; Hu, F. B.; Manson, J. E.; Rimm, E. B.; Willett, W. C. (2000). «Primary Prevention of Coronary Heart Disease in Women through Diet and Lifestyle». New England Journal of Medicine (en inglés) 343 (1): 16-22. Doi: 10.1056/NEJM200007063430103. PMID 10882764.

Hu., F., Manson, J., Stampfer, M., Graham, C., et al. (2001). Diet, lifestyle, and the risk of type two diabetes mellitus in women. The New England Journal of Medicine, 345(11), 790-797. Retrieved October 5, 2006, from proQuest database.

«Exercise». Medical-dictionary.thefreedictionary.com. Citando Gale Encyclopedia of Medicine. Copyright 2008. Cita: «Strengthening exercise increases muscle strength and mass, bone strength, and the body's metabolism. It can help attain and maintain proper weight and improve body image and self-esteem. »

«WHO: Obesity and overweight». who.int. Archivado desde el original el 4 de junio de 2004. 
«Physical Activity and Exercise: The Wonder Drug. » American Association of Kidney Patients.

«The miracle drug. » National Center for Biotechnology Information.

A. Cornil, A. De Coster, G. Copinschi, J. R. M. Franckson (1965). «Effect of muscular exercise on the plasma level of cortisol in man». European Journal of Endocrinology.

Cohen S., Williamson G. M. (1991). «Stress and infectious disease in humans». Psychological Bulletin109: 5-24. doi:10.1037/0033-2909.109.1.5. PMID 2006229.

«Una dieta equilibrada y actividad física, factores clave en mejora de salud». Consultado el 18 de agosto de 2012.

«Ejercicio intenso activa 184 genes que protegen contra cáncer de próstata». Consultado el 19 de agosto de 2012.

Jason Menoutis, Ed.D. (2008). «Physical Activity and Health». Nasm Pro.

Silberner, Joanne (7 de junio de 2010). «100 Years Ago, Exercise Was Blended Into Daily Life». Npr.org. Consultado el 23 de noviembre de 2010.

Wislett, Ulrik; Ellingsen O, Kemi O. (julio de 2009). «High=Intensity Interval Training to Maximize Cardiac Benefit of Exercise Taining? ». Exercise and Sports Sciences Reviews37 (3): 139-146. Doi: 10.1097/JES.0b013e3181aa65fc. PMID 19550205.

Hanc, J. 1987. «You're Health behind the Runner's Euphoria. »I''Newsday, April 21, 1987, I'I' 11. Retrieved October 5, 2006, from proQuest database.

Bouchard, Claude; Ping An, Treva Rice, James S. Skinner, Jack H. Wilmore, Jacques Gagnon, Louis Perusse, Arthus S. Leon, D. C. Rao (1 de septiembre de 1999). «Familial aggregation of VO (2max) response to exercise training: results from the HERITAGE Family Study». Journal of Applied Physiology87 (3): 1003-1008. PMID 10484570. Consultado el 17 de julio de 2007.

Kolata, Gina (12 de febrero de 2002). «Why Some People Won't Be Fit Despite Exercise». The New York Times. Consultado el 17 de julio de 2007.

Brutsaert, Tom D.; Esteban J. Parra (2006). «What makes a champion? Explaining variation in human athletic performance» (PDF). Respiratory Physiology \&Neurobiólogo151 (2-3): 109-123. doi:10.1016/j.resp.2005.12.013. PMID 16448865. Archivado desde el original el 10 de agosto de 2007. Consultado el 17 de julio de 2007.

Geddes, Linda (28 de julio de 2007). «Superhuman». New Scientist. pp. 35-41.

«Being active combats risk of functional problems». 
Gallego Antonio, J. \& Aguilar Parra, J. \& Cangas Díaz, A. \&Torrecillas, J. \& Justo, C. \& Mañas, I. «Programa de Natación Adaptada para personas mayores dependientes: Beneficios Psicológicos, Físicos y Fisiológicos.» (19 de diciembre de 2011). Revista de Psicología del Deporte, 2012. Vol. 21, núm. 1, pp. 125-133. ISSN1132-239X. Recuperado el 10 de marzo de 2012.

Márquez Sara, (1995). «Beneficios Psicológicos de la Actividad Física.». Rev. De Psicol. Gral. y Aplic.,48 (1): 185-206. «Existe mucha literatura acerca de los beneficios de la práctica de la actividad física vigorosa tales como, por ejemplo: mejora la autoconfianza, sensación de bienestar, satisfacción sexual, disminución de la ansiedad, disminución de la depresión o la mejora del funcionamiento intelectual».

Folkins, Carlyle H.; Sime, Wesley E. (1981). «Physical fitness training and mental health». American Psychologist36 (4): 373-389. Doi: 10.1037/0003-066X.36.4.373.

C B Taylor, J F Sallis, and R Needle (1985). «The relation of physical activity and exercise to mental health». Public Health Rep.100 (2): 195-202.

Kenneth R Fox (1999). «The influence of physical activity on mental well-being». Public Health Nutrition: 411ᄀ-418. doi:10.1017/S1368980099000567

\section{【L Ciencia}




\section{PARA CITAR EL ARTÍCULO INDEXADO.}

González E., \& Días J. (2018). Uso del ejercicio físico con fines profilácticos en el Adulto Mayor. Anatomía Digital, 1(2), 6-14 https://doi.org/10.33262*/visionariodigital.v3i4.949

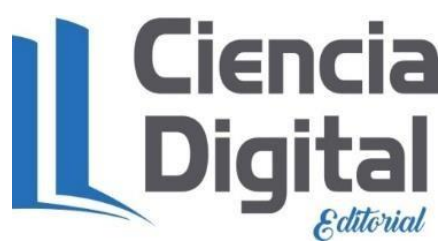

El artículo que se publica es de exclusiva responsabilidad de los autores y no necesariamente reflejan el pensamiento de la Revista Anatomía Digital.

El artículo queda en propiedad de la revista y, por tanto, su publicación parcial y/o total en otro medio tiene que ser autorizado por el director de la Revista Anatomía Digital.
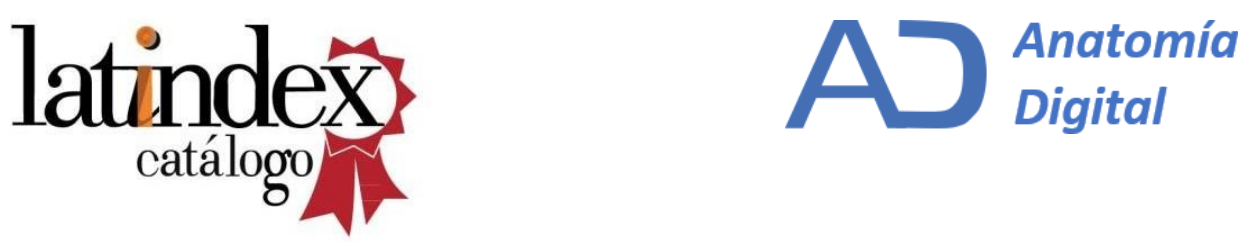